\title{
A Numerical Study on Impact of Taiwan Island Surface Heat Flux on Super Typhoon Haitang (2005)
}

\author{
Hongxiong Xu \\ State Key Laboratory of Severe Weather, Chinese Academy of Meteorological Sciences, Beijing 100081, China \\ Correspondence should be addressed to Hongxiong Xu; dorn1984@163.com
}

Received 4 March 2015; Revised 18 June 2015; Accepted 28 June 2015

Academic Editor: Antonio Donateo

Copyright (C) 2015 Hongxiong Xu. This is an open access article distributed under the Creative Commons Attribution License, which permits unrestricted use, distribution, and reproduction in any medium, provided the original work is properly cited.

\begin{abstract}
Three to four tropical cyclones (TCs) by average usually impact Taiwan every year. This study, using the Developmental Tested Center (DTC) version of the Hurricane WRF (HWRF) model, examines the effects of Taiwan's island surface heat fluxes on typhoon structure, intensity, track, and its rainfall over the island. The numerical simulation successfully reproduced the structure and intensity of super Typhoon Haitang. The model, especially, reproduced the looped path and landfall at nearly the right position. Sensitive experiments indicated that Taiwan's surface heat fluxes have significant influence on the super Typhoon Haitang. Compared to sensible heat (SH) fluxes, latent heat $(\mathrm{LH})$ is the dominant factor affecting the intensity and rainfall, but they showed opposite effects on intensity and rainfall. LH (SH) flux of Taiwan Island intensified (weakened) Typhoon Haitang's intensity and structure by transferring more energy from (to) surface. However, only LH played a major role in the looped path before the landfall of the Typhoon Haitang.
\end{abstract}

\section{Introduction}

TCs are one of the most dangerous natural hazards to human society and the environment [1-4]. Severe TCs produce destructive winds, high surges, torrential rains, and severe floods, usually resulting in serious property damage and loss of life $[3,5-8]$. Thus, it is of great interest to accurately predict TC's track and intensity. However, although the skill of position (track) forecasts has increased significantly, the accuracy of intensity forecasts has still to be improved [914]. Apparently, tropical cyclone intensity change interweaves multiscale nonlinear interactions among different physical processes, which are often poorly resolved or parameterized by global and even regional models [15-17]. Such processes include surface fluxes of moisture and heat, moist convection, radiative transfer, and cloud microphysics $[13,18]$.

Surface fluxes of moisture and heat are critical for mesoscale meteorological models [13, 19]. Modeling studies of sensitivity of TC development and intensity to sea/land surface conditions [20-22] were performed through alterations of the exchange coefficients of heat, moisture, and momentum at the surface. These studies revealed the relation between tropical cyclones development and maintenance of the model storm. The air-sea exchange of $\mathrm{SH}$ appears to be far less important. The studies of Tuleya and Kurihara [23] indicate that suppression of evaporation is the most important factor in the decay of a storm upon landfall. A reasonable change in surface temperature, moisture, and distribution of surface roughness can make a considerable difference in behavior [24].

Three to four tropical cyclones (TCs) by average usually impact Taiwan every year. The aforementioned studies emphasize role of surface conditions of land and ocean in affecting TCs, and the effect of surface heat fluxes of Taiwan Island is the issue that still needs to be addressed. In this study, we aimed to extend these previous studies by numerical simulations of Typhoon Haitang to explore the influence of surface heating of Taiwan Island on typhoon behavior. Super Typhoon Haitang was selected because of its cyclonic looping motion around Taiwan and strong intensity.

The track deflection of super typhoon Haitang was simulated in Jian and $\mathrm{Wu}$ [25] utilizing the advanced research version of the Weather Research and Forecasting model (WRF) with a $4 \mathrm{~km}$ fine mesh. It is found that the northerly advection flow results in a sharp southward turn of the westward-moving storm. The advection wind vectors rotate 
cyclonically in time and well match the motion of the simulated vortex during the looping process. Lowering the Taiwan terrain elevations to $70 \%$ or $40 \%$ of those in the control experiment reduces the southward track deflection and loop amplitude. Reducing elevation to $10 \%$ does not show a looping track and thus demonstrates the key role of the terrain-induced channeling effect.

Despite the work in Jian and $\mathrm{Wu}$ [25], the role of surface heat fluxes over Taiwan Island to Haitang remains to be investigated. In this study, some numerical experiments are designed to examine the track, intensity, and rainfall over Taiwan associated with surface heat fluxes over the island in two particular aspects. One is the role of heat fluxes of Taiwan Island in the intensity and structure of Haitang, and the other is its impact on the cyclonic looping motion of Super Typhoon Haitang before its landfall in Taiwan, which poses a rather unique scientific and forecasting problem [25]. The rest of the paper is organized as follows. Section 2 describes the overview of Typhoon Haitang. Sections 3 and 4 show the model description and experiment design. The results of simulations and the detailed effects of heat fluxes on intensity, rainfall, and track were presented in Section 5. Section 6 gives summary.

\section{Overview of Typhoon Haitang}

Super typhoon Haitang developed from a poorly organized depression at UTC 10 July 2005 . By 0000 UTC 12 , it had reached tropical storm $\left(17 \mathrm{~m} \mathrm{~s}^{-1}\right)$ and upgraded to typhoon $\left(32.7-41.4 \mathrm{~m} \mathrm{~s}^{-1}\right)$ at 1800 UTC 13. It turned westsouthwestward after 0600 UTC 12 and west-northwestward 3 days later heading toward Taiwan Island, largely steered by the easterlies associated with the subtropical high to its north [26]. By 06 UTC July 16, it strengthened into a super typhoon $\left(\geqslant 51.0 \mathrm{~m} \mathrm{~s}^{-1}\right)$ and became a threat to Taiwan Islands. Before making landfall in Taiwan at 0000 UTC 18, Haitang experienced sharp southward in its track and a loop to the east of Taiwan.

\section{Model Description}

The Hurricane Weather Research and Forecast System (HWRF) Model [27, 28], an advanced hurricane prediction system based on Weather Research and Forecast software framework [29], was developed at NCEP's Environmental Modeling Center (EMC) for understanding the hurricane forecast issues and improvement of hurricane prediction. This HWRF modeling system, based on the Nonhydrostatic Mesoscale Model dynamic core [30, 31], is a highresolution coupled air-sea-land prediction model with a movable nested grid capable of following the moving TC [27]. The configuration of the HWRF system consists of outermost (parent) domain and two movable nested grids with resolutions of 27,9 , and $3 \mathrm{~km}$, respectively, and 42 vertical levels. This HWRF modeling system has been used for real-time operational forecasts at NCEP since 2007 and was made accessible to research community in April 2010. The rotated latitude-longitude projection is utilized to maximize

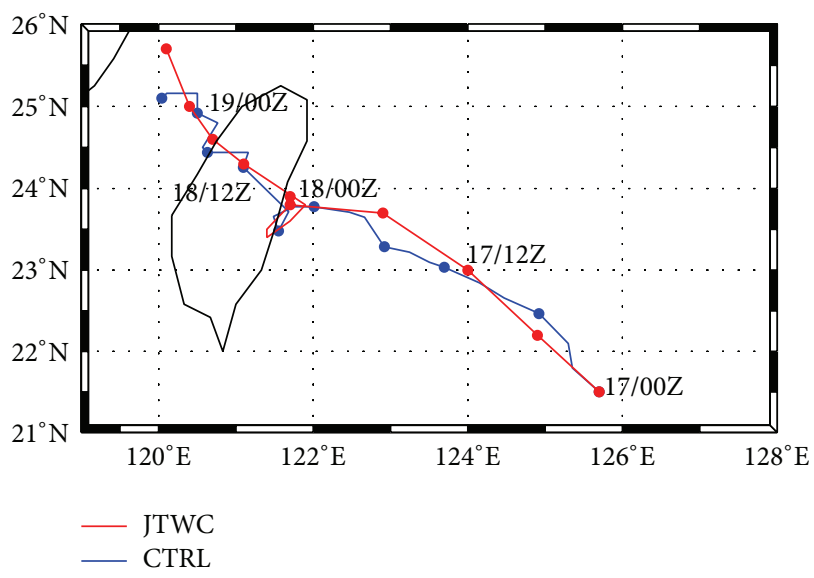

Figure 1: Comparison of the best track (red) and the simulated track (blue) of Typhoon Haitang from CTRL experiment. Each dot denotes Haitang's central position every $6 \mathrm{~h}$.

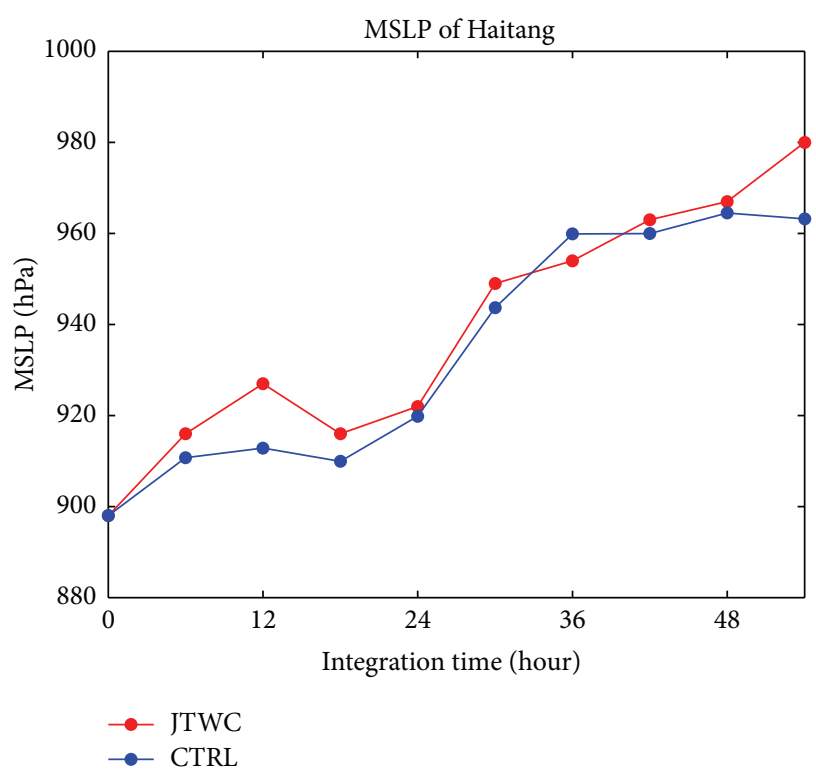

FIgure 2: Time series of the minimum sea level pressure (MSLP, units of hPa) from the 6-hourly JTWC best track analysis (red) and CTRL simulation (blue) during the period of 0000 UTC 17 July to 0600 UTC 19 July 2005.

efficiency. The discretization grid is based on the Arakawa-E grid. The parameterization schemes are briefly described in references $[27,28,32]$.

HWRF uses a TC initialization and relocation algorithm to initialize the TC based on the observed position and intensity. The Gridpoint Statistical Interpolation (GSI), a 3Dimensional Variational (3DVAR) Data Assimilation (DA) system, is implemented for initializing the environmental circulation outside the TC vortex area. The vortex initialization, relocation, and GSI DA produce the initial conditions to better match the actual TC observations. Therefore, a composite synthetic vortex, which is generated from bogus vortex or cycled from the vortex of the previous cycle, is assimilated in the observed TC location. The initialization of the HWRF 

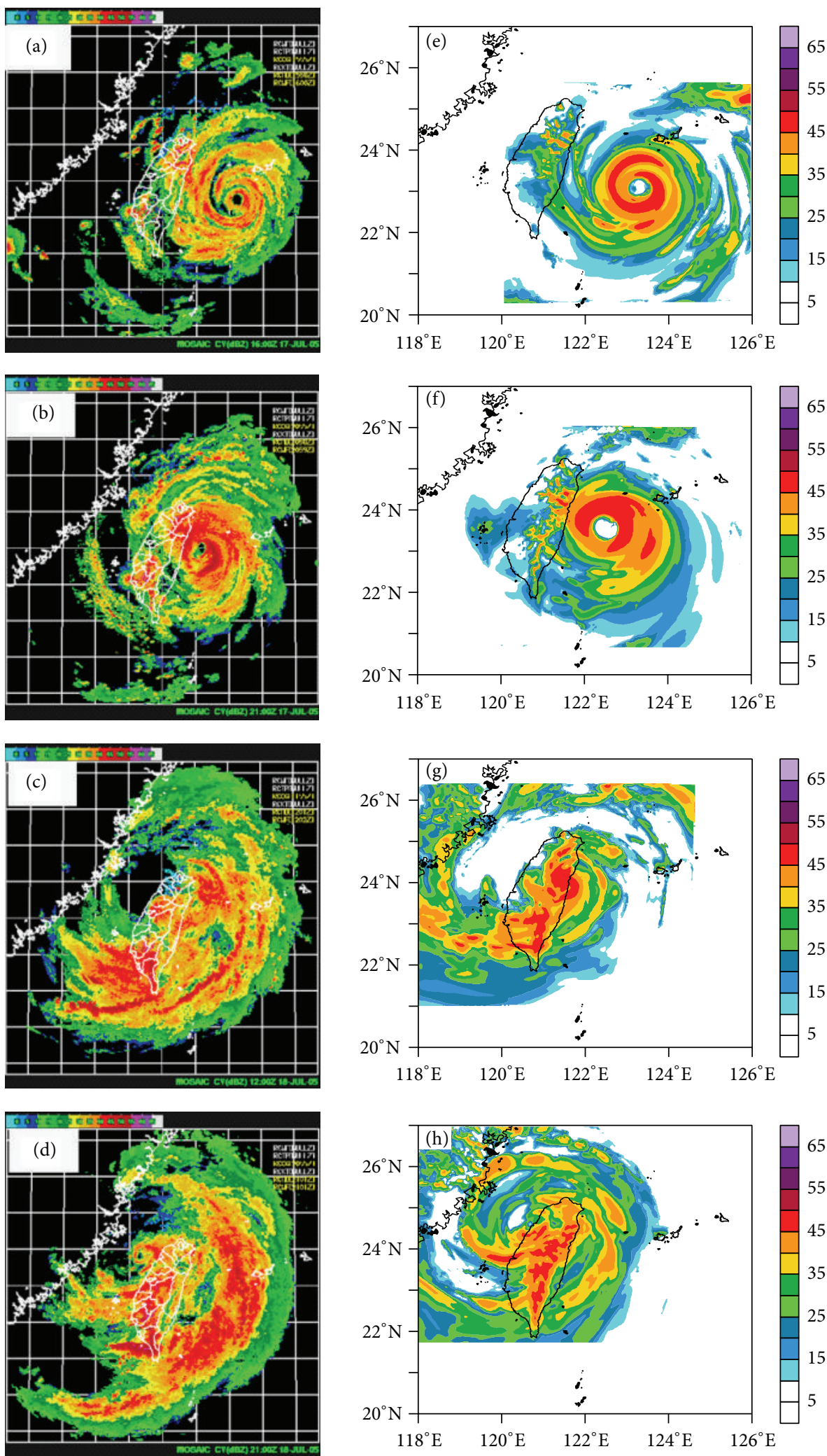

Figure 3: Radar reflectivity (unit: dbz) from Taiwan Central Weather Bureau at (a) 1600 UTC 17 July 2005, (b) 2100 UTC 17 July 2005 , (c) 1200 UTC 18 July 2005, and (d) 2000 UTC 18 July 2005, and radar reflectivity (unit: dbz) from the $3 \mathrm{~km}$ CTRL simulation at (e) 1500 UTC 17 July 2005 (f) 2100 UTC 17 July 2005, (g) 1200 UTC 18 July 2005, and (h) 2100 UTC 18 July 2005. 


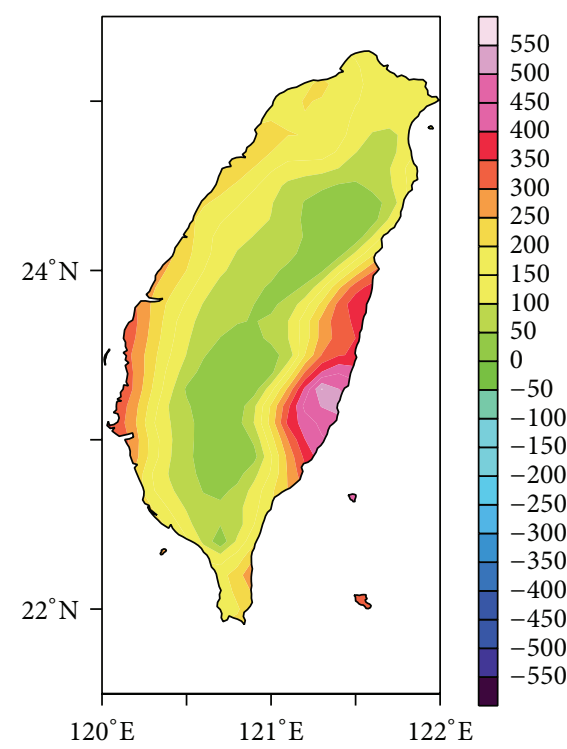

(a) Latent heat

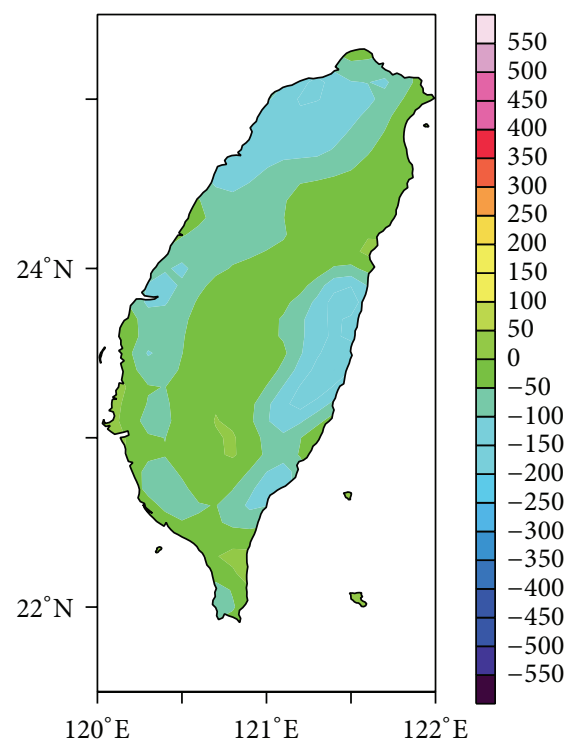

(b) Sensible heat

Figure 4: Simulated surface (a) LH flux $\left(\mathrm{W} \mathrm{m}^{-2}\right)$ and (b) SH flux $\left(\mathrm{W} \mathrm{m}^{-2}\right)$ averaged over $54 \mathrm{~h}$ integration of Taiwan Island.

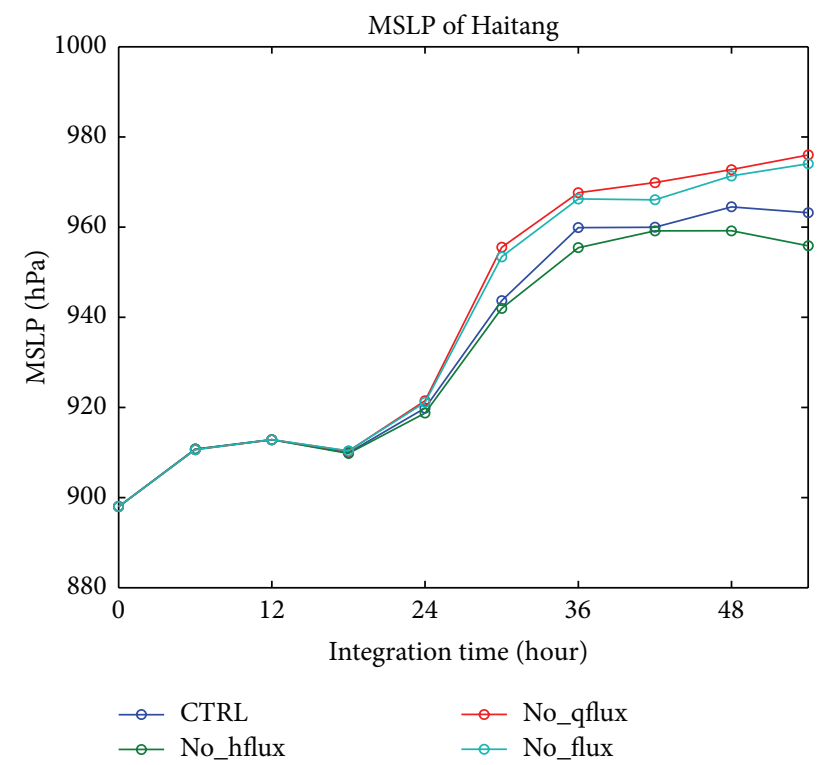

Figure 5: Time series of the modeled minimum central sea level pressure (hPa) of each experiment from 0000 UTC 17 July to 0600 UTC 19 July 2005.

model consists of four major steps: (1) create first guess fields from the Global Forecast System (GFS) analysis or reanalysis (such as NCEP/NCAR reanalysis); (2) remove the TC vortex from the global analysis; (3) add the synthetic vortex through the vortex initialization procedure; and (4) assimilate any available observational data outside TC core. Steps (3) and (4) provide major advantages over the conventional GFDL (Geophysical Fluid Dynamics Laboratory) TC initialization procedure.

\section{Experimental Design}

A series of $54 \mathrm{~h}$ integration experiments are carried out to explore the impact of Taiwan Island heat flux on Haitang. NCEP/NCAR FNL reanalysis [33] is used as initial and boundary conditions. The CTRL simulation starts from 0000 UTC 17 July 2005 with bogus vortices based on the vortex initialization as above. After this initialization method, the initial analyses of the minimum sea level pressure of Haitang is $898 \mathrm{hPa}$, which is nearly the same as the best-track analyses from the Joint Typhoon Warning Center (JTWC) at 0000 UTC 17 July 2005. Sensitivity experiments are performed the same as CTRL but removing LH in No_qflux, SH in No_hflux, and both SH and LH in No_flux experiment over Taiwan Island.

\section{Result}

Figure 1 shows comparison of the best track and the simulated track (CTRL) of Typhoon Haitang. Haitang initially moves northwestward to Taiwan and then turns suddenly southward with a cyclonic looping path before landfall. It makes landfall around 0600 UTC 18 July and leaves at about 1800 UTC 18 July. The CTRL experiment reproduces reasonably the cyclonic looping path and the time of landing in and leaving Taiwan. Figure 2 compares the TC intensity between the control experiment and JTWC analysis. Such a comparison indicates that the simulated intensity agrees well with that from JTWC during most of the integration period, despite of an underestimated intensification rate for the $6 \sim 18 \mathrm{~h}$ of simulated time. In general, control experiment shows good track simulations for Haitang. The entire simulation in CTRL shows the fairly accurate track and intensity evolutions.

Figure 3 shows the horizontal distributions of reflectivity from the radar observation and the $3 \mathrm{~km}$ CTRL simulation. 


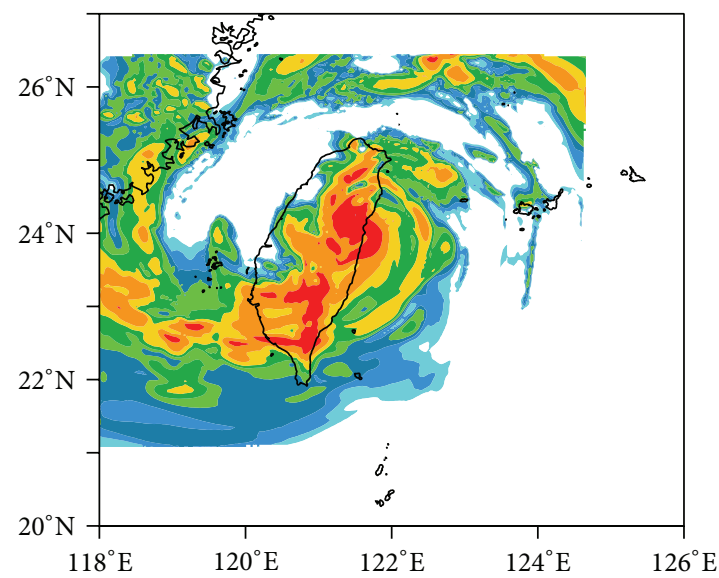

(a)

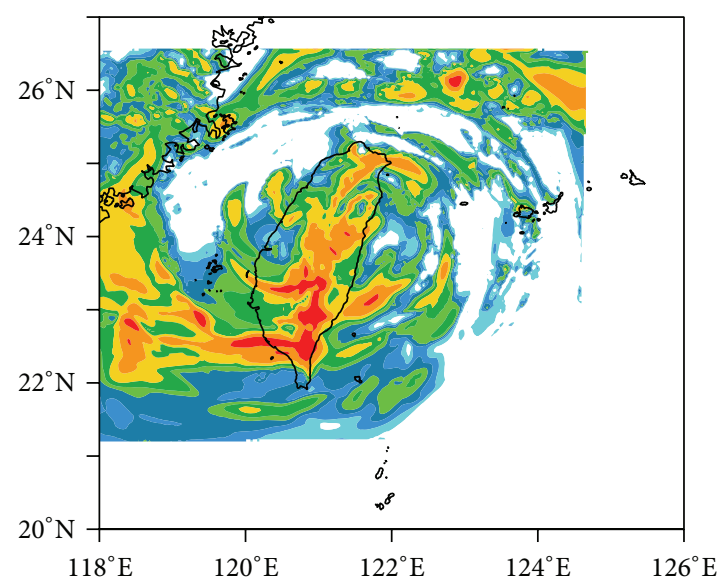

(c)
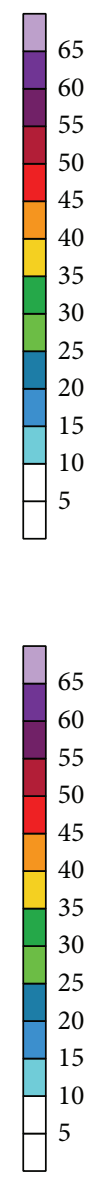

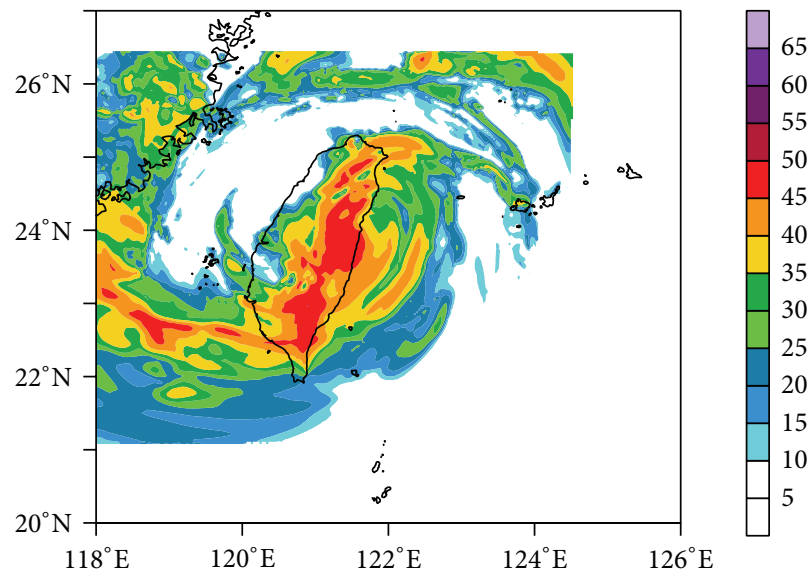

(b)

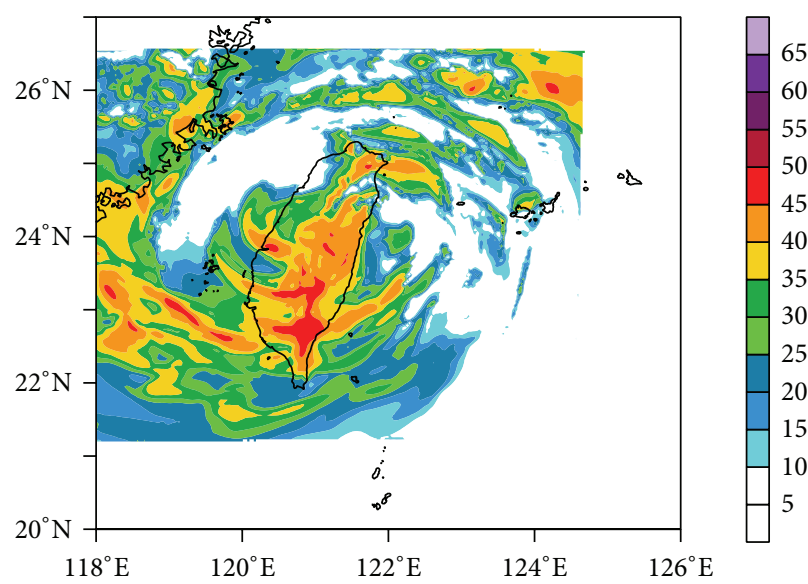

(d)

FIGURE 6: Horizontal maps of the simulated radar reflectivity from (a) CTRL, (b) No_hflux, (c) No_qflux, and (d) No_flux at 1200 UTC 18 July 2005.

The model simulation reproduced the observed typhoon structure reasonably well, including eye, eyewall, and rainbands. The azimuthal phase of the simulated rainbands, especially, matches well that of the observation. Since the CTRL reproduced reasonably well Haitang's intensity, structure, and movement, the CTRL simulation is studied to understand the impact of Taiwan Island surface heat flux on the intensity and structure of and the looping motion of Haitang.

Taking an average over the period of model integration (Figure 4), the LH fluxes are positive over the Taiwan Island. In contrast, $\mathrm{SH}$ fluxes are negative over most of Taiwan, indicating an opposite direction of the surface energy transport between the typhoon and the boundary. The highest values of the LH flux are found in the east and west sides, while the lowest are in the central area. Similarly, distribution of the SH flux resembles that of the LH flux.

5.1. Impact of Heat Fluxes on the Typhoon Intensity. Figure 5 shows MSLP of the sensitivity experiments and the control run. It shows that both the LH flux (Exp. No_qflux) and the SH flux (Exp. No_hflux) have an effect on typhoon intensity. The result in Exp. No_qflux illustrates that LH flux from the Taiwan Island is favorable for typhoon maintenance.
Without considering the effect on the LH flux of Taiwan, Typhoon Haitang would be weakened. This might be related to increased TC energy transported from the island surface and enhanced gain of energy when the typhoon made landfall over Taiwan. In contrast, Typhoon Haitang lost energy and attenuated during landfall. In short, Haitang is attenuated in No_qflux but deepens in No_hflux. Taking integration results until $54 \mathrm{~h}$, the MSLP in Exp. No_hflux (without $\mathrm{SH})$ is decreased by $7.3 \mathrm{hPa}$ while it increased by 12.8 and $10.8 \mathrm{hPa}$ in Exp. No_qflux and Exp. No_flux, respectively. These results indicate that heating fluxes from Taiwan affect the development of super typhoon Haitang with opposite impact.

To evaluate the impact of Taiwan surface heat fluxes on the typhoon, it is also meaningful to compare radar reflectivity from sensitivity simulations. Figure 6 reveals that all the simulations, including the CTRL, produce some rainbands from south of the storm to its north. It is important to note that the highest differences among the experiments are seen north of Taiwan. As radar reflectivity is a variable that is sensitive to the hydrometeor variables, whose structure and intensity changes are closely related to tropical cyclone intensity [34], the difference of radar reflectivity may be 


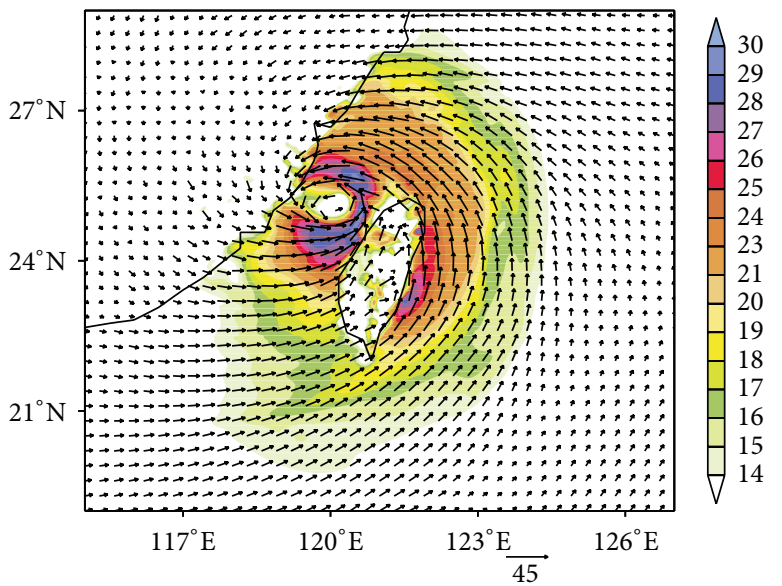

(a)

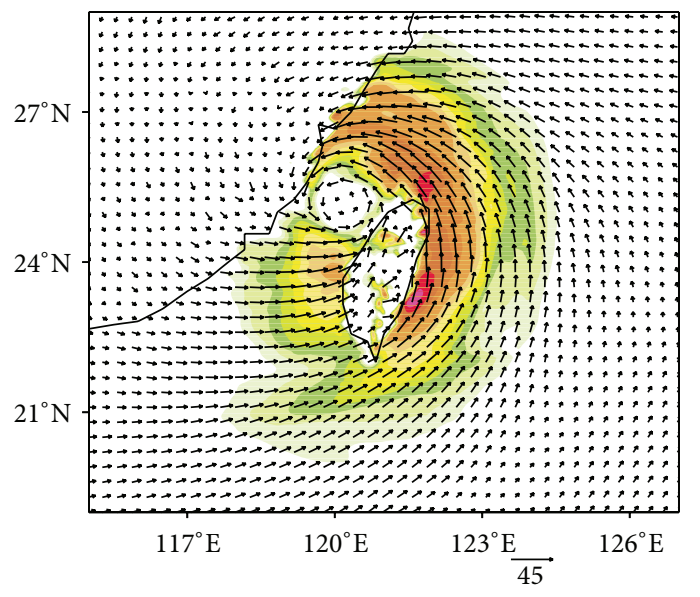

(c)

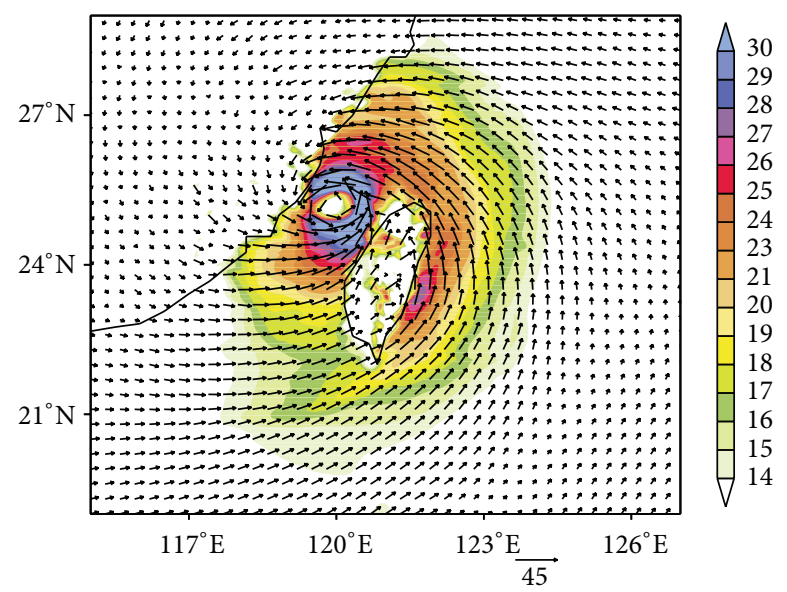

(b)

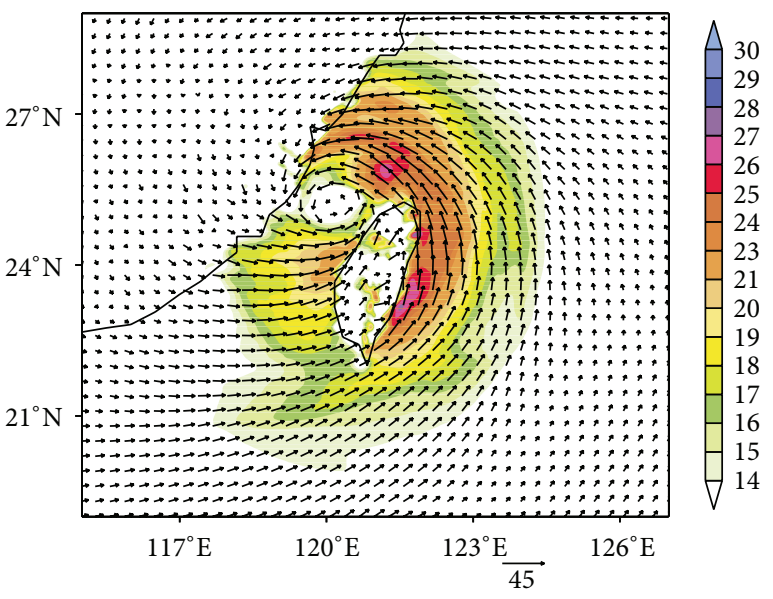

(d)

Figure 7: 10 m wind (units: $\mathrm{m} \mathrm{s}^{-1}$ ) for (a) CTRL, (b) No_hflux, (c) No_qflux, and (d) No_flux at 0600 UTC 19 July 2005.

employed to represent the extent of typhoon intensity. By comparing Exp. No_hflux and CTRL (Figures 6(a) and 6(b)), it is shown that they both show high dbz near typhoon center over north of Taiwan, but No_hflux shows slightly stronger spiral rainbands at the southwest quadrant. The result in Exp. No_qflux reveals that weakening of Haitang intensity is significant without inclusion of LH fluxes. Typhoon Haitang weakened in No_flux nearly the same as No_qflux, indicating that LH fluxes play a more important role in development of the typhoon.

Simulated $10 \mathrm{~m}$ wind field at 0600 UTC 19 July 2005 (Figure 7) is consistent with results above, that is, the $\mathrm{LH}$ $(\mathrm{SH})$ trend to weaken (strengthen) wind at typhoon center (Figures 7(b) and 7(c)). Figure 8 shows the West-east cross sections of equivalent potential temperature at 1200 UTC 18 July 2005. The result from the CTRL experiment shows that an area of high $\theta_{e}$ appeared from surface to $100 \mathrm{hPa}$ over the typhoon center. Low $\theta_{e}$ areas located at the west and east of the typhoon center. The gradient of the $\theta_{e}$ is large between two low areas and the typhoon center, indicating strong upward movement in the area of the typhoon eyewall [14]. Such distribution of $\theta_{e}$ suggests the warm and wet structure of typhoon [5]. Comparatively, No_hflux shows larger gradient, as a result of the cutoff of $\mathrm{SH}$ fluxes from typhoon to the island surface, which is favorable for sustaining of large gradient $\theta_{e}$ structure of typhoon. By contrast, without LH in No_qflux (Figure 8(c)), the gradient is lower than that in CTRL. No_flux (Figure 8(d)) also shows the same trend as No_qflux.

By comparing the $54 \mathrm{~h}$ accumulated rainfall over Taiwan produced from the sensitivity experiments and the CTRL run (Figure 9), it is found that the transfer of both latent and sensible heat fluxes over Taiwan Island has an impact on the intensity of typhoon rainfall (Figures 9(b), 9(c), and 9(d)). Without the latent heat effect (Figure 9(c)), typhoon precipitation would be reduced significantly over north of the Taiwan Island but increased to the south. The area averaged precipitation decreased from $344 \mathrm{~mm}$ in CTRL to $306 \mathrm{~mm}$ in No_qflux and increased to $347 \mathrm{~mm}$ in No_hflux over Taiwan Island. SH show little impact on the island (Figure 10). From the sensitivity experiments, it is suggested that the LH flux plays a dominant role in intensity of typhoon rainfall.

5.2. Impact of Heat Fluxes on the Typhoon Track. Figure 11 shows MSLP of the modeled TCs. Typhoon Haitang showed an asymmetric structure, when it is crossing Taiwan. High 


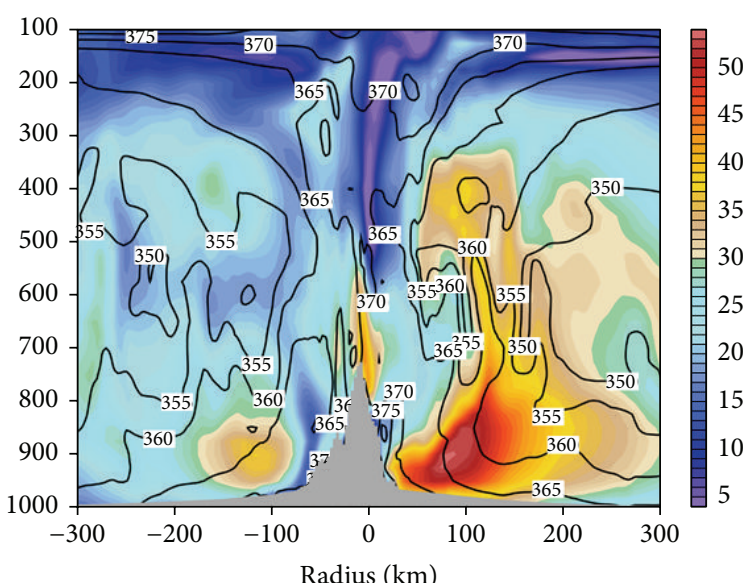

(a)

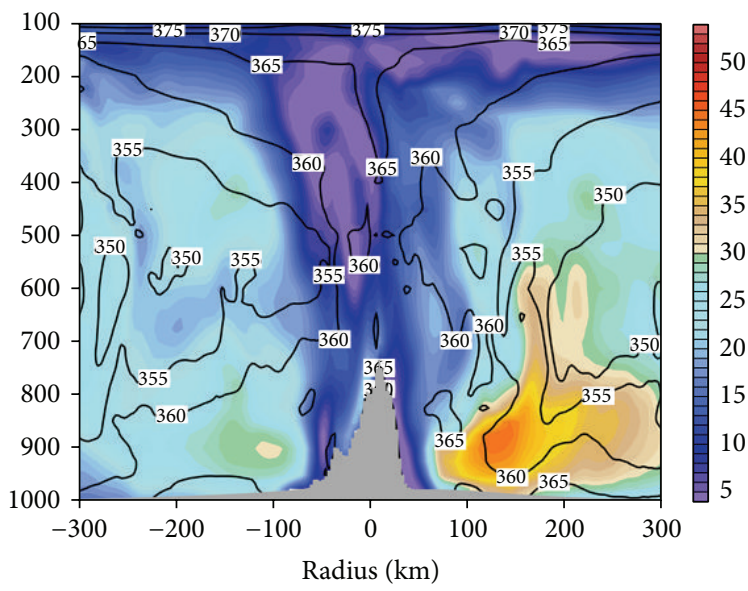

(c)

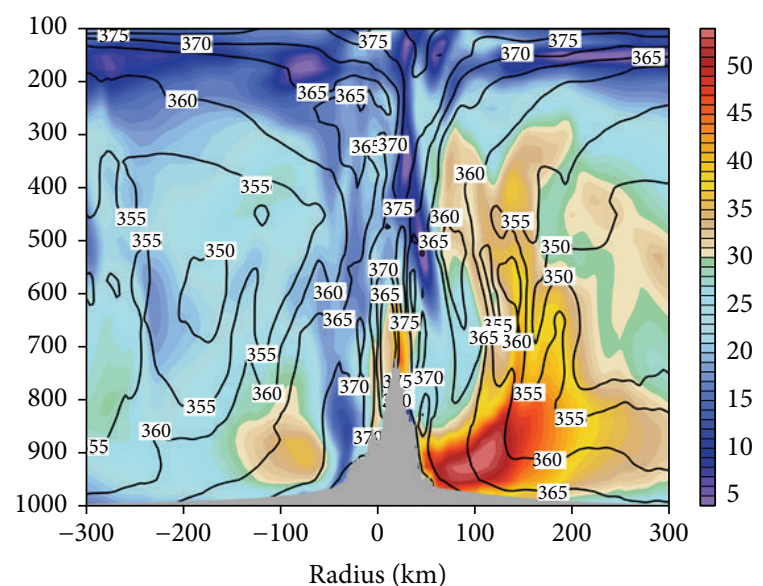

(b)

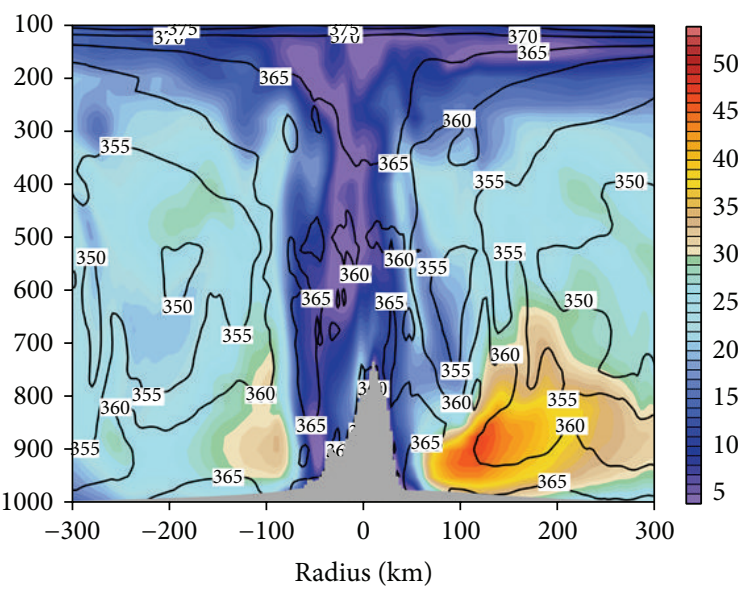

(d)

Figure 8: West-east cross sections of equivalent potential temperature ( $\theta_{e}$, units: K) from (a) CTRL, (b) No_hflux, (c) No_qflux, and (d) No_flux at intervals of $3 \mathrm{~K}$, superposed with wind velocity (units: $\mathrm{m} \mathrm{s}^{-1}$ ) at 1200 UTC 18 July 2005.

values of $\mathrm{LH}$ and $\mathrm{SH}$ were both located at nearly the same position as Haitang landfall (Figure 4). Both the LH flux (Exp. No_qflux) and the SH flux (Exp. No_hflux) have a significant effect on the structure of Typhoon Haitang. However, Haitang is attenuated in No_qflux but deepens in No_hflux. It indicates that heating fluxes from Taiwan affect the structure of typhoons, which significantly influenced the looped track of Haitang. Without LH flux, the intensity of Haitang is attenuated and less asymmetric at 06 UTC 18 July 2005. Finally, the dominant part of the typhoon moved from east of Taiwan to northwest and changed its track. In short, LH flux of Taiwan Island plays an important role in the structure of Haitang, resulting in the looping motion.

Sensitivity experiments are also conducted to demonstrate the impact of the heat flux on the track of Haitang. By comparing EXP No_hflux, No_qflux, and No_flux, it shows that surface heat flux has significant influence on the track of Haitang, especially during the period of landfall. In No_hflux, the Taiwan SH is removed, but the storm still shows a looped path before landfall in Taiwan (Figure 12(a)); it deviates to southward during the landfall period. In No_qflux and No_flux (Figures 12(b) and 12(c)), the looped path disappeared indicating that track of Haitang is more sensitive to LH flux.

\section{Summary}

In this study, a mesoscale numerical model particularly designed for the purpose of hurricane research and forecast, HWRF, is used to quantify the influence of Taiwan Island's heat flux on the track and structure of Typhoon Haitang that made landfall at Taiwan. The HWRF system consists of two movable nested grids with resolutions of 27,9 , and $3 \mathrm{~km}$. CTRL experiment is performed with NCEP/NCAR FNL reanalysis. The experiment provided a reasonable replication of the track of typhoon Haitang during the $54 \mathrm{~h}$ integration, especially reproducing the looping motion east of Taiwan and the landfall location. The overall evolution of intensity also compared favorably with observations, although it underestimates intensification rate for the $6 \sim 18 \mathrm{~h}$ of simulated time. Furthermore, radar reflectivity was very similar to what was observed. The model simulation reproduced the observed typhoon structure reasonably well, including eye, eyewall, and rainbands. 


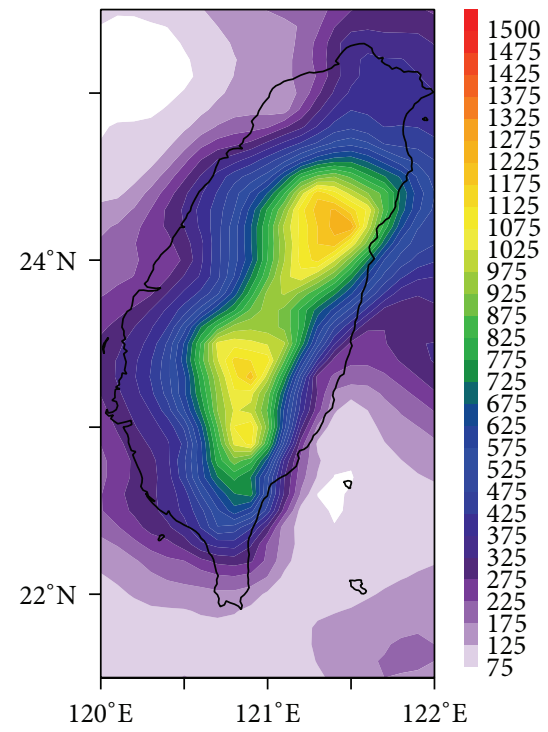

(a)

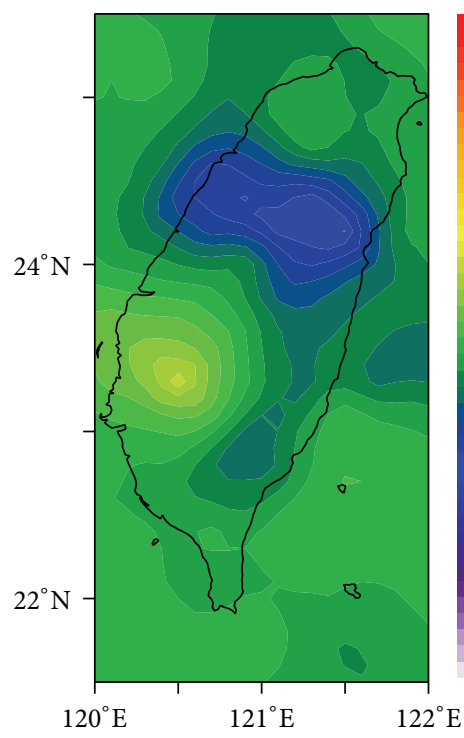

(c)

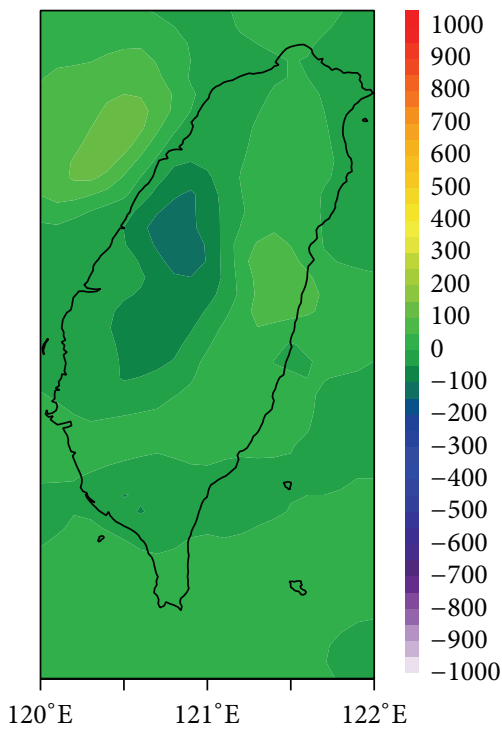

(b)

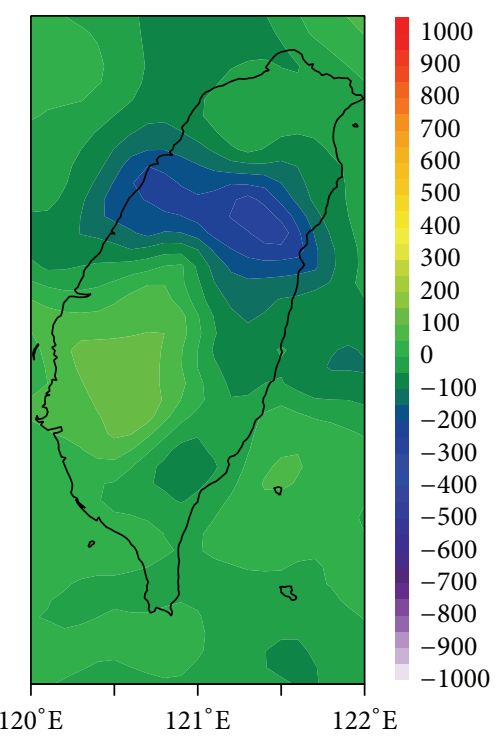

(d)

FIgURE 9: (a) Horizontal maps of the $54 \mathrm{~h}$ accumulated rainfall (units: mm) over Taiwan from CTRL experiment during 0000 UTC 17 July to 0600 UTC 19 July 2005; (b) differences of the $54 \mathrm{~h}$ accumulated rainfall (units: $\mathrm{mm}$ ) between No_hflux and CTRL experiment; (c) and (d) are the same as (b) but for No_qflux and No_flux.

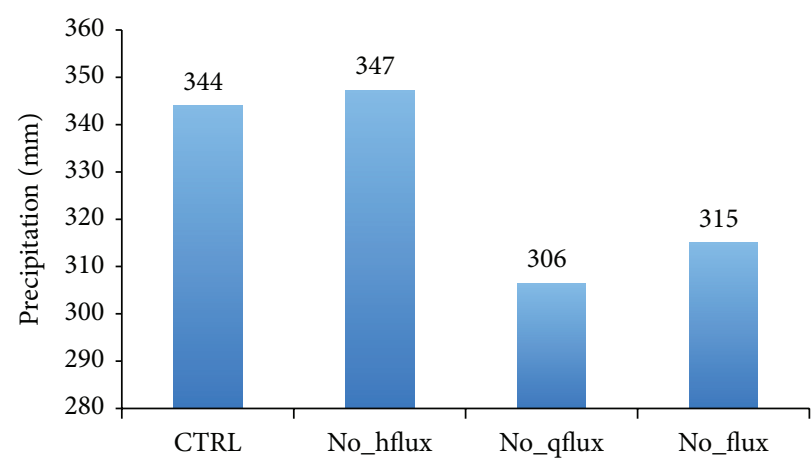

FIGURE 10: Area averaged $54 \mathrm{~h}$ accumulated rainfall (units: $\mathrm{mm}$ ) calculated over Taiwan Island. 


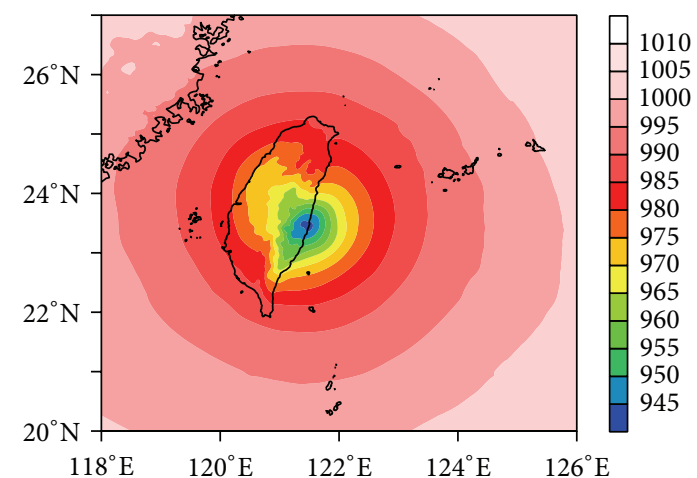

(a)

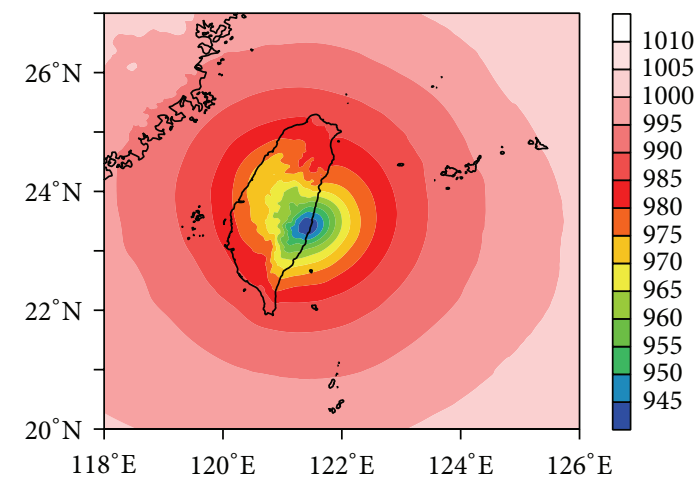

(b)

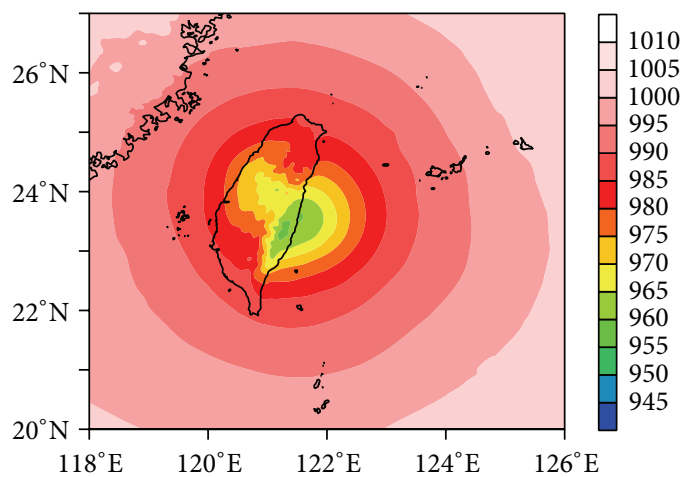

(c)

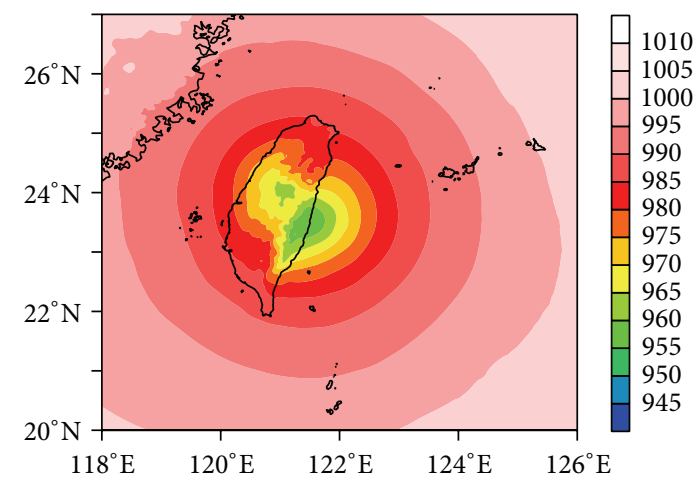

(d)

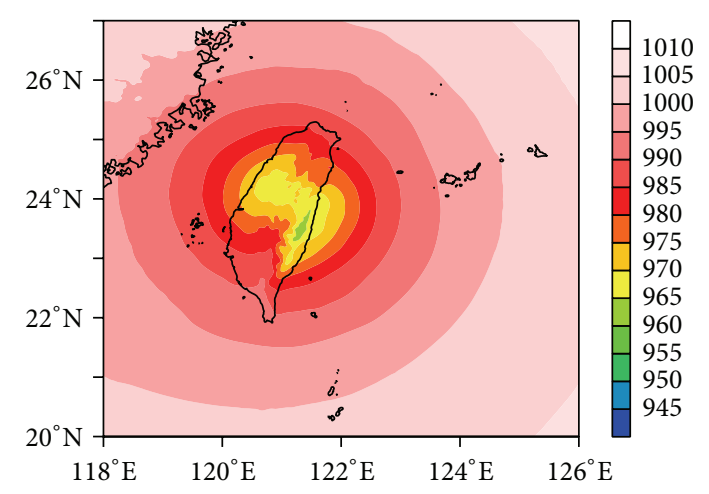

(e)

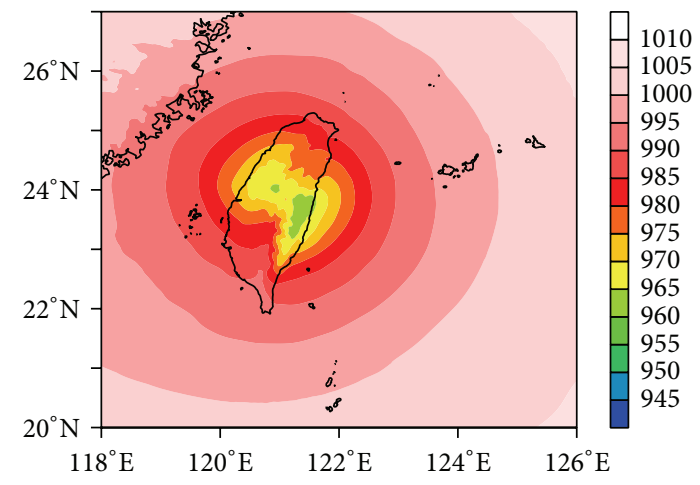

(f)

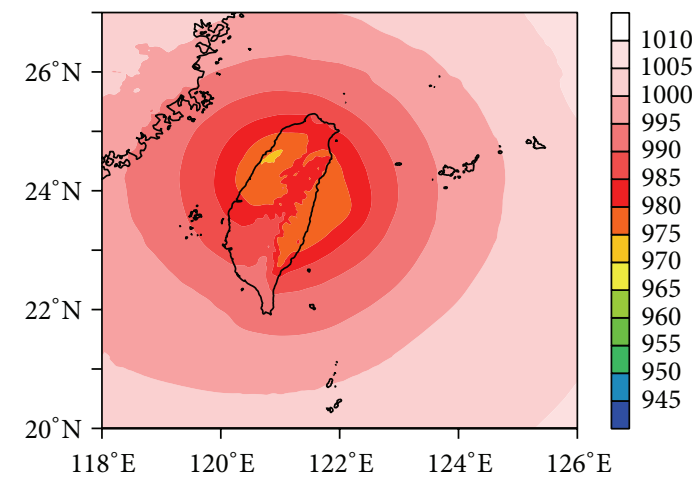

(g)

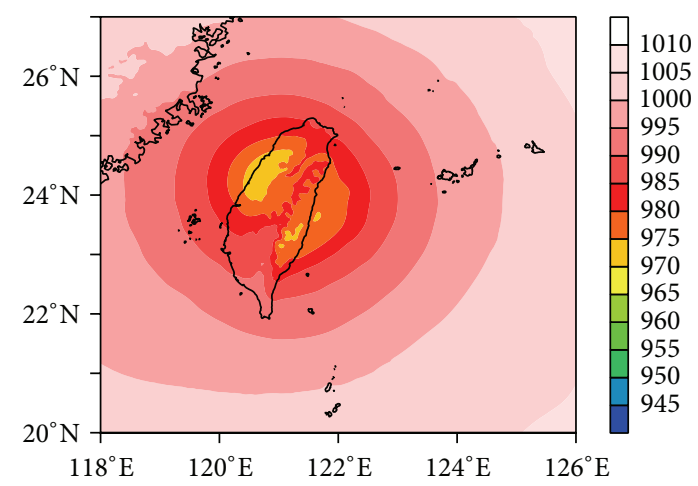

(h)

Figure 11: MSLP (units: hPa) of the modeled TC in (a) CTRL, (b) No_hflux, (c) No_qflux, and (d) No_flux at 06 UTC 18 July 2005; (e) (h) are the same as (a) (d) but for 12 UTC 18 July 2005. 


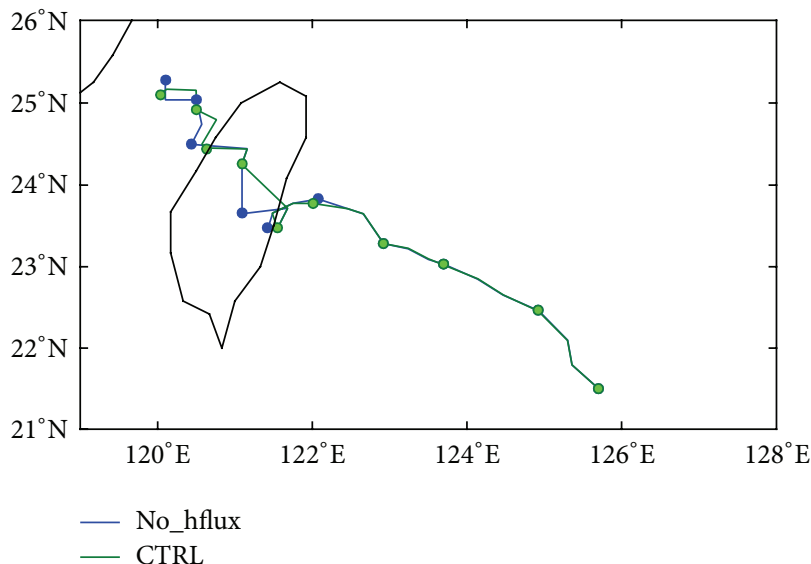

(a)

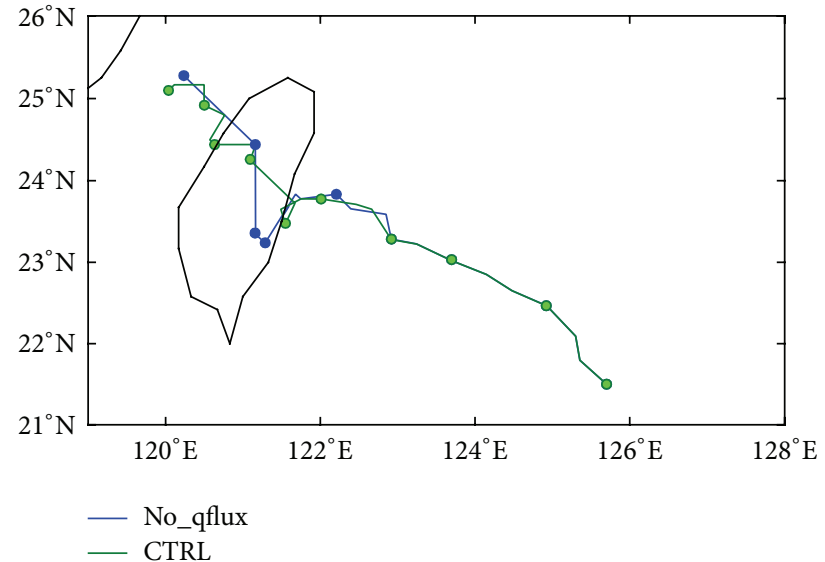

(b)

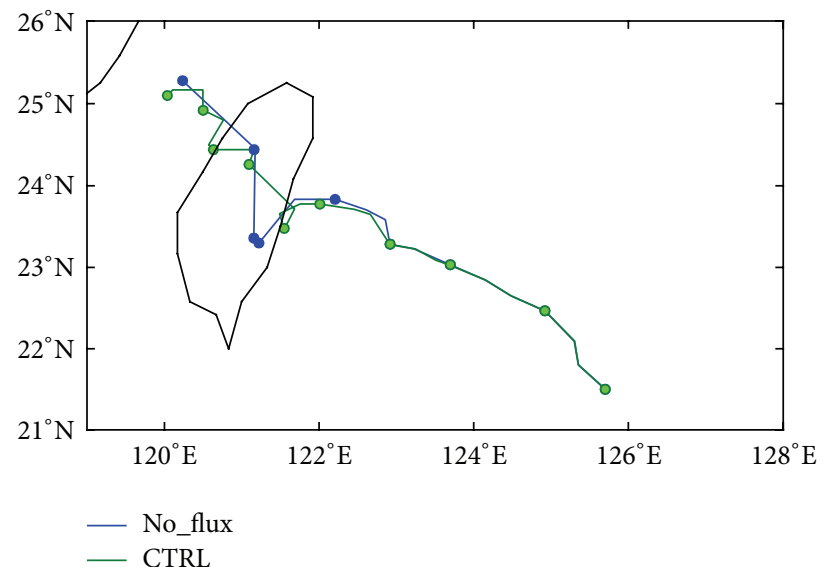

(c)

Figure 12: As in Figure 1, but for (a) No_hflux, (b) No_qflux, and (c) No_flux.

The effects of the Taiwan Island were studied using sensitivity experiments. Sensitivity experiments artificially removed the LH effect in No_qflux, SH in No_hflux, and both $\mathrm{SH}$ and LH in No_flux experiment over Taiwan Island. Results show that both LH flux and SH flux have an effect on typhoon intensity. However, Haitang is attenuated in No_qflux but deepens in No_hflux. Results indicate that heating fluxes from Taiwan affect development of typhoons with opposite impact and LH transfer playing dominant roles.

It indicates that heating fluxes from Taiwan affect the structure of typhoons, which significantly influenced the looped track of Haitang. Without LH flux, the intensity of Haitang is attenuated and less asymmetric. LH flux was affecting Haitang during crossing Taiwan. Finally, the dominant part of the typhoon moved from east of Taiwan to northwest and changed its track. In short, LH flux of Taiwan Island plays an important role in the structure of Haitang, resulting in the looping motion.

The future work is intended to study several other loopedpaths around Taiwan and to summarize their common characteristics. Furthermore, we hope to reveal the roles of island surface heat flux played in the structure of TC. The goal of this research is to improve the understanding of and ability to predict anomalous track of typhoon around Taiwan Island.

\section{Conflict of Interests}

The author declares that there is no conflict of interests regarding the publication of this paper.

\section{Acknowledgments}

This study is supported by the State Key Program of National Natural Science Foundation of China (Grant no. 41130960). The author would like to thank all the reviewers and editors for their professional advice to improve the paper.

\section{References}

[1] H. Chen, D.-L. Zhang, J. Carton, and R. Atlas, "On the rapid Intensification of Hurricane Wilma (2005). Part I. Model prediction and structural changes," Weather and Forecasting, vol. 26, no. 6, pp. 885-901, 2011.

[2] Y. Wang, Y. Wang, and H. Fudeyasu, "The role of Typhoon Songda (2004) in producing distantly located heavy rainfall in Japan," Monthly Weather Review, vol. 137, no. 11, pp. 3699-3716, 2009.

[3] Y. Wang and C.-C. Wu, "Current understanding of tropical cyclone structure and intensity changes-a review," Meteorology and Atmospheric Physics, vol. 87, no. 4, pp. 257-278, 2004. 
[4] L. Chen, X. Xu, Z. Luo, and J. Wang, Introduction to Tropical Cyclone Dynamics, Meteorological Press, Beijing, China, 2002.

[5] L. Chen and Y. Ding, An Introduction to West Pacific Typhoons, Science Press, Beijing, China, 1979.

[6] C. Lianshou and L. Zhexian, "Effect of the interaction of different scale vortices on the structure and motion of typhoons," Advances in Atmospheric Sciences, vol. 12, no. 2, pp. 207-214, 1995.

[7] F.-C. Chien and H.-C. Kuo, "On the extreme rainfall of Typhoon Morakot (2009)," Journal of Geophysical Research: Atmospheres, vol. 116, no. 5, Article ID D05104, 2011.

[8] X. Ge, T. Li, S. Zhang, and M. Peng, "What causes the extremely heavy rainfall in Taiwan during Typhoon Morakot (2009)?" Atmospheric Science Letters, vol. 11, no. 1, pp. 46-50, 2010.

[9] E. N. Rappaport, J. L. Franklin, L. A. Avila et al., "Advances and challenges at the national hurricane center," Weather and Forecasting, vol. 24, no. 2, pp. 395-419, 2009.

[10] K. A. Emanuel, "Sensitivity of tropical cyclones to surface exchange coefficients and a revised steady-state model incorporating eye dynamics," Journal of the Atmospheric Sciences, vol. 52, no. 22, pp. 3969-3976, 1995.

[11] K. A. Emanuel, "An air-sea interaction theory for tropical cyclones. Part I: steady-state maintenance," Journal of the Atmospheric Sciences, vol. 43, no. 6, pp. 585-605, 1986.

[12] K. A. Emanuel and M. Živković-Rothman, "Development and evaluation of a convection scheme for use in climate models," Journal of the Atmospheric Sciences, vol. 56, no. 11, pp. 1766-1782, 1999.

[13] B. W. Green and F. Zhang, "Impacts of air-sea flux parameterizations on the intensity and structure of tropical cyclones," Monthly Weather Review, vol. 141, no. 7, pp. 2308-2324, 2013.

[14] Y. Li and L. Chen, "Numerical study on impacts of boundary layer fluxes over wetland on sustention and rainfall of landfalling tropical cyclone," Acta Meteorologica Sinica, vol. 63, no. 5, pp. 683-693, 2005.

[15] F. D. Marks and L. K. Shay, "Landfalling tropical cyclones: forecast problems and associated research opportunities," Bulletin of the American Meteorological Society, vol. 79, no. 2, pp. 305-323, 1998.

[16] B.-W. Shen, W.-K. Tao, W. K. Lau, and R. Atlas, "Predicting tropical cyclogenesis with a global mesoscale model: hierarchical multiscale interactions during the formation of tropical cyclone Nargis (2008)," Journal of Geophysical Research D: Atmospheres, vol. 115, no. 14, 2010.

[17] R. L. Elsberry, T. D. B. Lambert, and M. A. Boothe, "Accuracy of atlantic and eastern north pacific tropical cyclone intensity forecast guidance," Weather and Forecasting, vol. 22, no. 4, pp. 747-762, 2007.

[18] H. Chen and D.-L. Zhang, "On the rapid intensification of hurricane wilma (2005). Part II: convective bursts and the upper-level warm core," Journal of the Atmospheric Sciences, vol. 70, no. 1, pp. 146-162, 2013.

[19] A. Xiu and J. E. Pleim, "Development of a land surface model. Part I. Application in a mesoscale meteorological model," Journal of Applied Meteorology, vol. 40, no. 2, pp. 192-209, 2001.

[20] K. Ooyama, "Numerical simulation of the life cycle of tropical cyclones," Journal of the Atmospheric Sciences, vol. 26, no. 1, pp. 3-40, 1969.

[21] S. L. Rosenthal, "The response of a tropical cyclone model to variations in boundary layer parameters, initial conditions, lateral boundary conditions, and domain size," Monthly Weather Review, vol. 99, no. 10, pp. 767-777, 1971.
[22] W. Shen, I. Ginis, and R. E. Tuleya, "A numerical investigation of land surface water on landfalling hurricanes," Journal of the Atmospheric Sciences, vol. 59, no. 4, pp. 789-802, 2002.

[23] R. E. Tuleya and Y. Kurihara, "A numerical simulation of the landfall of tropical cyclones," Journal of the Atmospheric Sciences, vol. 35, no. 2, pp. 242-257, 1978.

[24] R. E. Tuleya, M. A. Bender, and Y. Kurihara, "A simulation study of the landfall of tropical cyclones," Monthly Weather Review, vol. 112, no. 1, pp. 124-136, 1984.

[25] G.-J. Jian and C.-C. Wu, "A numerical study of the track deflection of supertyphoon haitang (2005) prior to its landfall in Taiwan," Monthly Weather Review, vol. 136, no. 2, pp. 598615, 2008.

[26] H. Yu, W. Huang, Y. H. Duan, J. C. L. Chan, P. Y. Chen, and R. L. $\mathrm{Yu}$, "A simulation study on pre-landfall erratic track of typhoon Haitang (2005)," Meteorology and Atmospheric Physics, vol. 97, no. 1-4, pp. 189-206, 2007.

[27] S. Gopalakrishnan, Q. Liu, T. Marchok et al., Hurricane Weather Research and Forecasting (HWRF) Model Scientific Documentation, Development Tech Center, 2010.

[28] S. G. Gopalakrishnan, F. Marks Jr., X. Zhang, J.-W. Bao, K.S. Yeh, and R. Atlas, "The experimental HWRF system: a study on the influence of horizontal resolution on the structure and intensity changes in tropical cyclones using an idealized framework," Monthly Weather Review, vol. 139, no. 6, pp. 1762 1784, 2011.

[29] W. C. Skamarock, J. B. Klemp, J. Dudhia et al., "A description of the advanced research WRF version 3," NCAR Technical Note NCAR/TN-475+STR, 2008.

[30] Z. I. Janjic, J. P. Gerrity, and S. Nickovic, "An alternative approach to nonhydrostatic modeling," Monthly Weather Review, vol. 129, no. 5, pp. 1164-1178, 2001.

[31] Z. I. Janjic, "A nonhydrostatic model based on a new approach," Meteorology and Atmospheric Physics, vol. 82, no. 1-4, pp. 271285, 2003.

[32] K.-S. Yeh, X. Zhang, S. Gopalakrishnan et al., "Performance of the experimental HWRF in the 2008 Hurricane Season," Natural Hazards, vol. 63, no. 3, pp. 1439-1449, 2012.

[33] National Centers for Environmental Prediction (NCEP), NCEP FNL Operational Model Global Tropospheric Analyses, Continuing from July 1999, Research Data Archive at the National Center for Atmospheric Research, Computational and Information Systems Laboratory, Boulder, Colo, USA, 2000.

[34] X. Zou, Y. Wu, and P. S. Ray, "Verification of a high-resolution model forecast using airborne Doppler radar analysis during the rapid intensification of Hurricane Guillermo," Journal of Applied Meteorology and Climatology, vol. 49, no. 4, pp. 807-820, 2010. 

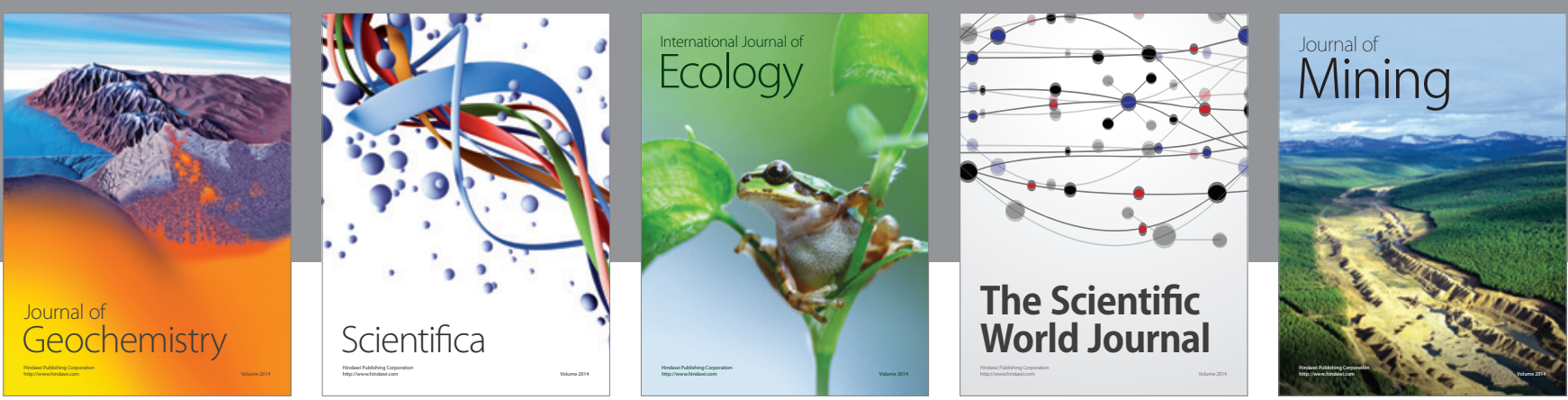

The Scientific World Journal
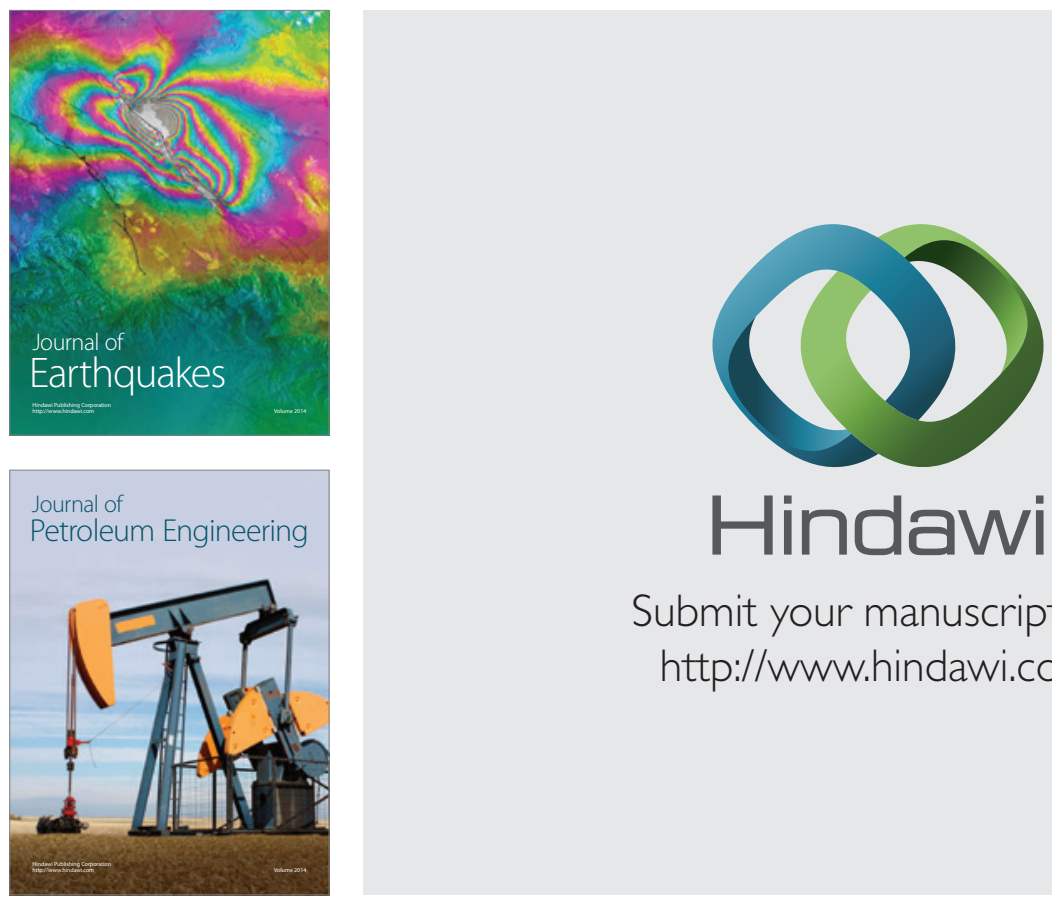

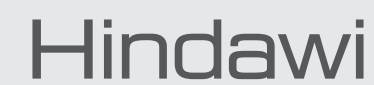

Submit your manuscripts at

http://www.hindawi.com
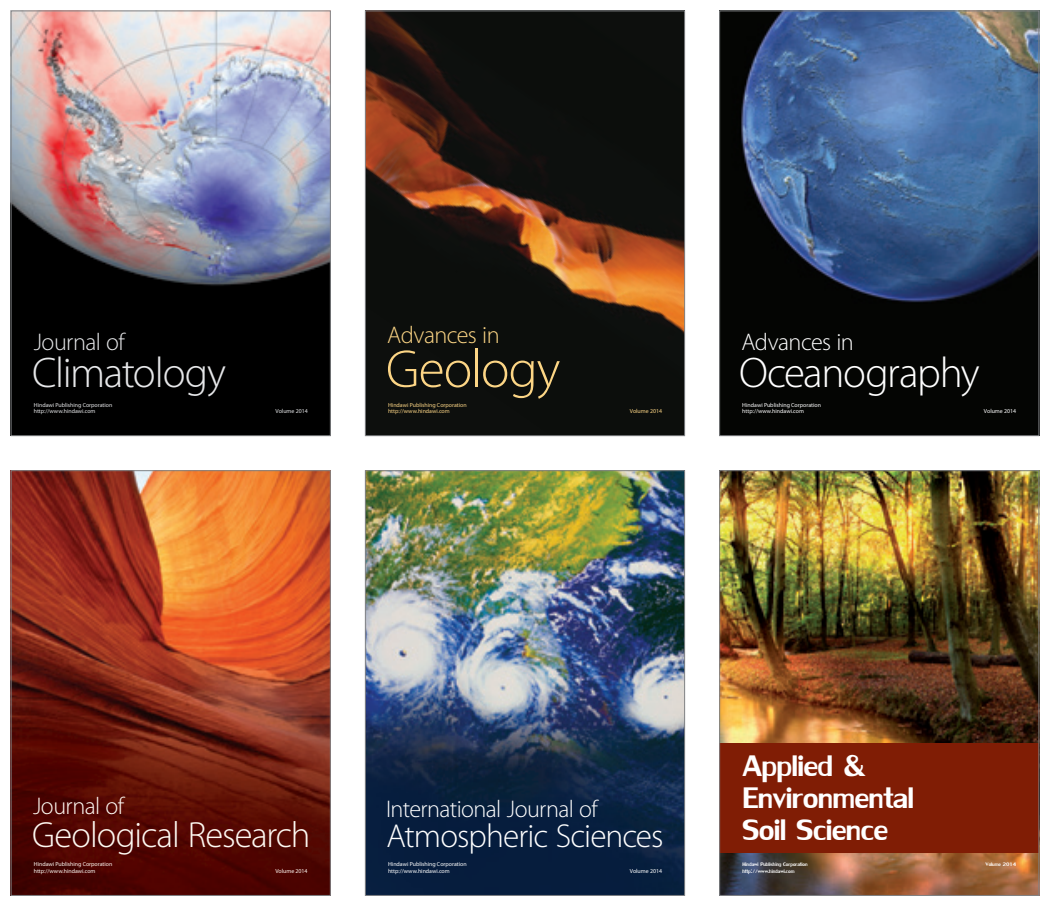
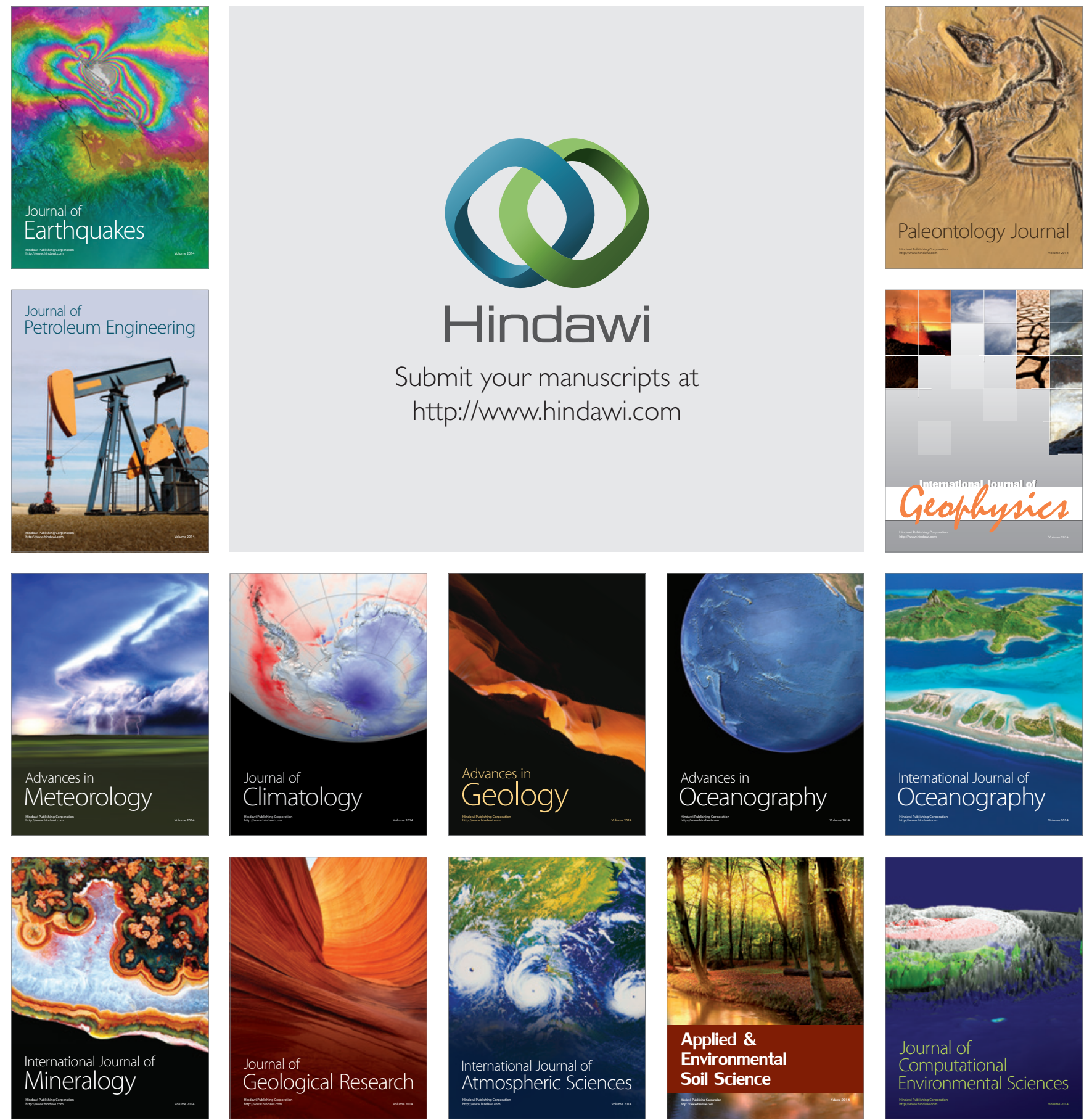